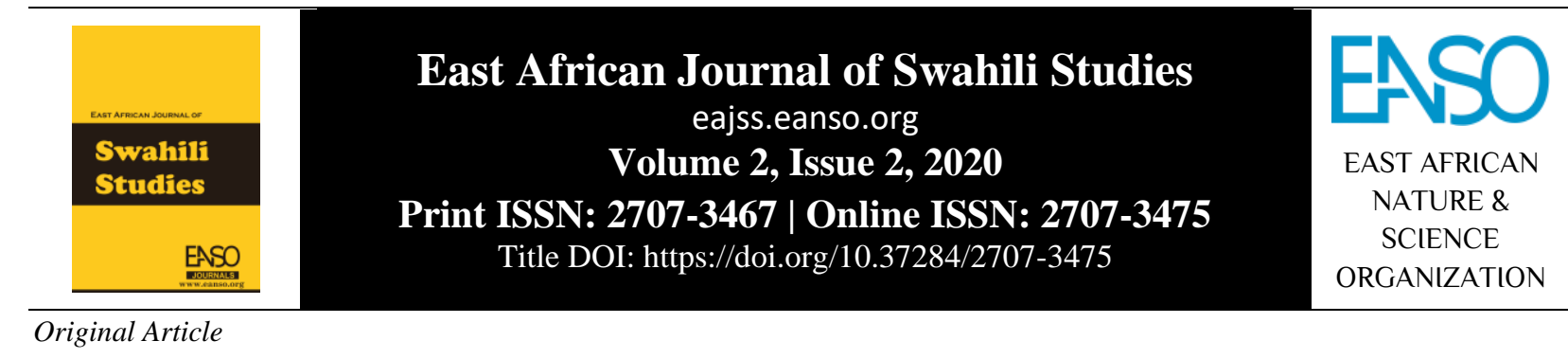

\title{
Nafasi ya Fasihi Katika Kuwasilisha Masuala ya Mazingira Kupitia Riwaya Teule Tikitimaji (2013) na Msimu wa Vipepeo (2006) za K. W. Wamitila
}

\author{
Esther Lucy Wesonga ${ }^{1}$, Dkt. Beverlyne Asiko Ambuyo ${ }^{2}$ \& Prof. Rocha Chimerah Mzungu ${ }^{3}$ \\ ${ }^{1}$ Chuo Kikuu cha Mlima Kenya, S.L.P. 342 - 01000, Thika, Kenya. \\ ${ }^{2}$ Chuo Kikuu cha Maseno, S.L.P. 3275 - 40100, Kisumu, Kenya. \\ ${ }^{3}$ Chuo Kikuu cha Pwani, S.L.P. 195 - 80108, Kilifi, Kenya. \\ *Barua pepe ya mawasiliano: lucyesther87@gmail.com
}

DOI ya Nakala: https://doi.org/10.37284/eajss.2.2.192

\section{Tarehe ya Uchapishaji: IKISIRI}

11 Agosti 2020 Suala la mabadiliko ya hali ya anga ni janga linaloendelea kukumba ulimwengu kwa jumla. Kutokuwepo kwa usawazishaji wa ekolojia ni jambo ambalo Istilahi Muhimu: linawatia shaka adinasi wengi. Fasihi ni kioo cha jamii na inapaswa kuwasilisha hali halisi ya maisha ya binadamu. Utafiti huu unachambua nafasi

Fasihi Mazingira, ya fasihi katika kuwasilisha masuala ya mazingira kupitia riwaya mbili teule za Tikitimaji, Wamitila, Tikitimaji (2013) na Msimu Wa Vipepeo (2006). Mwandishi huyu Msimu wa Vipepeo, Ekolojia. ameonyesha ari katika uwanja wa fasihi mazingira, kwenye makala yake ya "Place and placelessness in Kiswahili literature: The role of setting in Kiswahili creative writing" anasema kuwa mandhari huchukua nafasi katika kuwasilisha masuala ya mazingira. Kazi hii ilichunguza namna mwandishi alivyotumia fasihi kuwasilisha masuala ya mazingira katika riwaya zake za hivi karibuni. Ingawa kwa miaka mingi, masuala ya mazingira yamehusishwa na sayansi haswa katika kutoa takwimu kamilifu kuhusiana na masuala ya mazingira, fasihi inaendelea kuchukua dhima mpya katika jamii inayozidi kukua kiteknolojia. Utafiti huu uliongozwa na madhumuni yafuatayo: kubainisha jinsi mwandishi wa riwaya ya Tikitimaji alivyotumia wahusika ili kuwasilisha maudhui ya mazingira. kueleza mitindo ya lugha iliyotumiwa kuwasilisha masuala ya mazingira na tatu, kutathimini iwapo mwandishi amefaulu kuwasilisha maudhui ya mazingira riwayani. Utafiti huu ulitumia kiunzi cha nadharia kutoka kwa nadharia ya fasihi mazingira na nadharia ya uhalisia. Mihimili zitakazotumiwa kutoka nadharia ya uhalisia ni: kazi ya fasihi imejitosheleza, kazi ya msanii inapaswa kuwasilisha ukweli, mtunzi anaangalia matatizo na kuchunguza chanzo chake na mwisho wahusika ni vielelezo yakini vya binadamu wa kawaida kwa vile wao hutumia lugha ya kawaida 
wanayoitumia humwezesha mwanadamu kujifafanulia uwezo wake wa kutenda mambo. Mihimili ya fasihi mazingira iliyotumika ni: kuangalia dhana za ekolojia katika kazi za fasihi, mitindo na uwezo wa lugha na jinsi mitindo hiyo huathiri namna binadamu anavyotagusana na mazingira na mwisho binadamu hawezi ishi bila ekolojia lakini ekolojia huweza kuishi bila binadamu. Data ilipangwa kwenye makundi na kuchanganuliwa kwa kuzingatia madhumuni ya utafiti na mihimili ya kiunzi cha nadharia. Data iliwasilishwa kwa njia ya maelezo. Utafiti huu umelenga kuhamasisha wasomi na waandishi wa fasihi wajikite zaidi kulenga masuala ya mazingira ambayo ndicho kitovu cha maisha ya binadamu. Utafiti ulibaini kuwa fasihi ni nyenzo mwafaka ya kuwasilisha masuala ya mazingira kupitia vipengele vyake. Kwani huingiliana sana na utamaduni wa jamii.

\section{APA CITATION}

Wesonga, E., Ambuyo, B., \& Mzungu, R. (2020). Nafasi ya Fasihi Katika Kuwasilisha Masuala ya Mazingira Kupitia Riwaya Teule Tikitimaji (2013) na Msimu wa Vipepeo (2006) za K. W. Wamitila. East African Journal of Swahili Studies, 2(2), 54-64. https://doi.org/10.37284/eajss.2.2.192

\section{CHICAGO CITATION}

Wesonga, Esther, Beverlyne Ambuyo, and Rocha Mzungu. 2020. "Nafasi ya Fasihi Katika Kuwasilisha Masuala ya Mazingira Kupitia Riwaya Teule Tikitimaji (2013) na Msimu wa Vipepeo (2006) za K. W. Wamitila”. East African Journal of Swahili Studies 2 (2), 54-64. https://doi.org/10.37284/eajss.2.2.192.

\section{HARVARD CITATION}

Wesonga, E., Ambuyo, B. and Mzungu, R. (2020) "Nafasi ya Fasihi Katika Kuwasilisha Masuala ya Mazingira Kupitia Riwaya Teule Tikitimaji (2013) na Msimu wa Vipepeo (2006) za K. W. Wamitila”, East African Journal of Swahili Studies, 2(2), pp. 54-64. doi: 10.37284/eajss.2.2.192.

\section{IEEE CITATION}

E. Wesonga, B. Ambuyo, and R. Mzungu, "Nafasi ya Fasihi Katika Kuwasilisha Masuala ya Mazingira Kupitia Riwaya Teule Tikitimaji (2013) na Msimu wa Vipepeo (2006) za K. W. Wamitila”, EAJSS, vol. 2, no. 2, pp. 54-64, Aug. 2020.

\section{MLA CITATION}

Wesonga, Esther, Beverlyne Ambuyo, and Rocha Mzungu. "Nafasi ya Fasihi Katika Kuwasilisha Masuala ya Mazingira Kupitia Riwaya Teule Tikitimaji (2013) na Msimu wa Vipepeo (2006) za K. W. Wamitila”. East African Journal of Swahili Studies, Vol. 2, no. 2, August 2020, pp. 54-64, doi:10.37284/eajss.2.2.192.

\section{UTANGULIZI}

Utafiti huu ulichunguza nafasi ya fasihi katika kuwasilisha masuala ya mazingira kupitia riwaya teule ya Tikitimaji (2013) na Msimu Wa Vipepeo (2006) zake Wamitila. Fasihi ni sanaa inayotumia lugha, hujishughulisha na maisha ya binadamu, huathiri, hugusa au huacha athari fulani inayopatikana katika umbo ambalo linatambuliwa na jamii (Wamitila, 2004). Njogu na Chimerah (1999) wanaeleza kuwa fasihi huwasilisha hali, maingiliano na mikinzano miongoni mwa binadamu na mazingira. Fasihi huwasilisha maudhui yenye mguso kwa wanajamii kupitia kwa lugha ya mvuto. Msanii huchota malighafi yake kutokana na hali halisi inayomzunguka. Fasihi hufanya kazi kwa kutuwezesha kukabiliana na majanga ya maisha na wakati vile vile hutoa maarifa ya kujitambua na kusuluhisha majanga hayo ya ulimwengu kwa jumla. Maudhui ya mazingira yameorodheshwa kama baadhi ya maudhui ibuka katika fasihi. Hii ni kwa mujibu wa Njogu na Chimera (2007) katika makala yao ya Ufundishaji wa masuala ibuka kwa Kiswahili.

Ekolojia yetu imo katika hali ya hatari ya maangamizi kutokana na matendo ya kibinafsi ya wanadamu (IPCC, 2013). Matendo kama vile; ukataji wa miti, ujenzi wa nyumba katika maeneo oevu, ongezeko la teknolojia haribifu na ukosefu wa uongozi adilifu. Mwisho wa matukio haya ni ongezeko la joto hewa na kuvugutwa kwa sayari, 
magonjwa, vifo na kupungua kwa mazao hali inayopelekea makali ya njaa.

Utata huu umewasilishwa na idadi kubwa ya ripoti na makongamano mbalimbali yanayoangazia majanga ya kimazingira. Ripoti ya Umoja wa kimataifa-ya Office for the Coordination of Humanitarian Affairs (mkutano wa dharura), inasema kuwa majanga ya tabianchi yanazidi kuongezeka. Shirika la umoja wa kimataifa kuhusu mazingira na maendeleo (UNCED) lililofanyika Rio de Jeneiro June 14 (1992) linalojulikana kama ajenda 21 lilikuwa na madhumuni ya kusitisha athari za kimazingira zinazotokana na kudorora kwa mazingira. Pia juhudi hizi za uhifadhi wa mazingira zinabainishwa na Wangari Mathai (1977) alipozindua muungano wa Green Belt movement akihusisha wanawake katika upanzi wa miti.

Kulingana na ripoti ya Intergovernmental Panel on Climatic Change (IPCC, 2014) ambayo ilihusisha makundi matatu, inasema kuwa miongo miwili ya karne ya ishirini ilikuwa yenye ongezeko la joto hewa. Hali hii inachangiwa na athari chafu ya hewa, ukataji miti hali ambayo imepunguza gesi ya kaboni diooksidi. Majanga haya ya kimazingira hukumba kila mwanajamii bila kubagua maeneo wanamopatikana. Maamuzi yetu kuhusiana na uchafuzi wa mazingira ndio hutofautiana. Kwa mujibu wa Reuveny (2007), anasema kuwa watu huweza kuliangalia suala la uchafuzi wa mazingira kwa njia tatu: kwanza, wanaweza kukaa mahali pale pachafu bila kufanya chochote; pili, binadamu wanaweza kuzikubali changamoto za majanga ya kimazingira-na mwisho, wanaweza kuamua kuleta mabadiliko katika sehemu zilizoathirika.

Maamuzi haya kuhusiana na masuala ya kimazingira, humotishwa na ufahamu wa kuhamasishwa kwa wanajamii kuhusiana na suala hili. Fasihi ni nyenzo mmojawapo muhimu yenye kuleta mahamasisho hayo. Kama asemavyo Vasquez (1983. Kazi ya sanaa huathiri watu na inachangia katika kuhimiza au kupuuza dhana zao, maazimio yao na hata maadili yao, yana msukumo wa kijamii ambao huathiri watu kwa nguvu zake za kihisia na kiitikadi. Chembilecho Vasquez (1983) fasihi huchukua nafasi kubwa sana katika kukuza maadili na mielekeo ya wanadamu kuhusiana na masuala fulani katika jamii. Ingawa mashirika ya kimazingira yamejihusisha sana na masuala ya mazingira katika viwango vya kitaifa na kimataifa, je, fasihi inahusika vipi katika kuwasilisha masuala yaya haya?

Greg (2007),anaeleza kwamba matatizo ya kimazingira yanaonekana kimsingi kuwa matatizo ya kisayansi na mijadala mingi kuhusu ongezeko la joto hewa huhusisha zaidi wanaangahewa kuliko watunzi wa ushairi au hata wahakiki wa fasihi. Kutokana na mwelekeo wake, masuala haya ya kimazingira yaweze kuwasilishwa kwa jamii kupitia fasihi kwani fasihi ndiyo huingiliana pakubwa na utamaduni wa jamii. Utafiti unachambua jinsi mwandishi wa riwaya hizi mbili, Tikitimaji (2013) na riwaya ya Msimu Wa Vipepeo(2007) anavyowasilisha maudhui haya. Wamitilia (1999), katika makala yake ya Place and placelessness in Kiswahili literature: The role of setting in Kiswahilili creative writing, anaangalia nafasi ya mandhari katika kuwasilisha masuala ya mazingira katika riwaya ambapo anasema kuwa mandhari huweza kutumika kuelezea hali ya mazingira ijayo. Anaonyesha ari katika uwanja huu wa fasihi mazingira ndiposa 111

bua riwaya zake za hivi karibuni ili kutathimini jinsi anavyowasilisha masuala haya ya mazingira. $\mathrm{Ni}$ muhimu suala la mazingira kuangaliwa kwa jicho pevu kwani binadamu wanapoharibu mazingira, utamaduni wao husambaratika. Kama asemavyo Ngugi (1995) msingi wa jamii ya binadamu ni ardhi, bila ardhi hamna jamii. Ngugi anaiona fasihi kama ung'amuzi wa mtunzi katika viwango vya kibinafsi na kijamii. Maoni yake Ngugi (1995) ni mkabala sawa na Unerchesky (1973) anayebaini kuwa ni muhimu fasihi ipongeze watu kwa mchango wao wanaofanya katika jamii.

Jinsi mazingira yanavyobadilika ndivyo fasihi inaendelea kuchukua dhima ya uchunguzi wa jamii huku ikibeba silaha mpya za utunzi, fikra za majadiliano na pia ikijihusisha na matatizo ya jamii mpya (Gromov, 2004). Katika kazi hizi za fasihi wahusika, mitindo na maudhui, hutumiwa kuwasilisha uhalisia wa kijamii. Suala la mazingira ni suala la kijamii na hivyo lapaswa kuangaziwa kwa undani kwani linapojitokeza katika fasihi huwasilisha maoni ya wanajamii. Uhusiano kati ya fasihi na mazingira ndio uliosababisha kuchipuka kwa uwanja wa fasihi ekolojia. Kama 
anavyofafanua fasihi mazingira, Rueckert (1978) katika makala yake yalioangazia matumizi ya ekolojia na dhana za ekolojia katika kazi za kifasihi. Fasihi ekolojia hunuia kuleta mageuzi ya kijamii na kimazingira kupitia fasihi. Mazingira hayarejelei tu mazingira asilia bali pia huhusisha mandhari yaliyojengwa na binadamu pamoja na mitagusano ya elementi hizo asilia na utamaduni. Uwanja huu unachukua mtazamo wa kiardhi. Uwanja huu upo mintarafu ya maisha ya binadamu. Pia umechanganuliwa kupitia diskosi aliali kuhusu mandhari, sehemu za dunia, spishi za viumbe hai, maliasili, na uzuifu wa uchafuzi wa viwandani. Kinachomtia ari mwandishi wa fasihi ekolojia ni hatua zake za kuzoa uhalisia wa dhana za kimazingira (Greg, 2004). Kwa hivyo, fasihi ekolojia ni usomaji wa matini za kifasihi unaoegemea kwenye masuala ya kimazingira. Upo uhusiano mkubwa kati ya fasihi na mazingira.

\section{SUALA LA UTAFITI}

Fasihi ni kioo cha jamii yetu. Fasihi huelimisha, hufundisha na huzindua jamii. Inafaa kuteua maudhui yenye mguso kwa jamii na kuyasimulia kwa kiwango cha juu cha umbuji. Majanga ya mazingira yanaendelea kuongezeka hata kama yanawekwa wazi na takwimu za kisayansi. Katika mwaka wa 2001, kamati ya IPCC ilitoa ripoti spesheli kuhusu utafiti wa hivi karibuni ambao uliacha mwanya finyu kitashwishi kuhusu mabadiliko ya anga. Mabadiliko haya yamesababishwa na matendo ya binadamu. Kwa mujibu wa World Conservation Union, takribani viumbe asili 103 wamepotea tangu mwaka wa 1800. Idadi hii ni mara hamsini ikilinganishwa na kiwango cha kupotea kiasilia. Takwimu hizi zinatokana na utafiti wa mwaka wa 1998 uliohusisha wanachama 400 wa taasisi ya sayansi na bayolojia. Tatizo tunalochunguza katika utafiti huu ni nafasi ya fasihi katika kuwasilisha masuala ya mazingira kupitia riwaya mbili teule za Tikitimaji (2013) na Msimu Wa Vipepeo (2007). Kama asemavyo Wafula na Njogu (2007), fasihi ni usanii ambao hauwezi kujitenga na mazingira, msanii mara nyingi huzoa malighafi yake ya sanaa katika jamii yake, lengo lake likiwa ni kuarifu kuhusu ulimwengu na jamii kwa jumla

\section{MAPITIO YA MAANDISHI}

Adamson na Slovic (2009) katika makala yao wanachambua zaidi upangaji wa fasihi mazingira katika mikondo miwili, wa kwanza na wa pili. Wanasema kuwa mkondo mpya na ambao ni watatu, ni wa kutambua maadili na uhusika wa taifa huku ikipita mipaka ya kikabila na ya kitaifa. Mkondo huu unaangalia sura zote za uzoevu wa binadamu. Nia yao ni kuangazia majanga ya kimazingira na jinsi yanavyoathiri watu maskini na watu wa rangi tofauti tofauti. Utafiti huu ndio msingi wa fasihi mazingira na jinsi inavyojihusisha na masuala ya kijamii na kisiasa katika nchi za kimagharibi. Utafiti huu Ulibainisha tabia ya wahusika katika kuchangia uchafuzi wa mazingira katika riwaya teule za Wamitila. Vilevile, unaangalia maudhui ya mazingira kama yanavyowasilishwa katika riwaya mahususi za Wamitila, Tikitimaji(2013) na Msimu Wa Vipepeo (2007).

Nixon na Ronald (2014) walishughukia vitabu na mashairi yafuatayo: Riwaya ya Nakuruto (2009) iliyoandikwa na Momanyi. Riwaya hii inaangazia suala ya ufeministi wa kiekolojia. Waandishi walimtumia mhusika Nakuruto kuhamasisha jamii kuhusiana na uharibifu wa mazingira. Katika hali ya kukomboa mazingira, mhusika Nakuruto anapitia changamoto nyingi sana haswa kwenye jamii yenye mifumo ya kiubabe-dume. Nixon na Ronald (2009) wanasema kuwa mhusika Nakuruto katika riwaya hii anasukumwa na sheria ya UNFCC (2009) ya uwajibikaji wa pamoja. Sheria hizi za UNFCC msingi wake unatokana na utafiti wa kisayansi ambao haukuhusisha asasi za kitamaduni. Mwandishi anakubaliana na Moore (2008) kuwa upo uhusiano wa moja kwa moja kati ya mazingira na mwanamke. Hata hivyo, utafiti huu unashughulikia masuala ya mazingira na uhusiano wao na wanawake. Utafiti wetu unatathmini nafasi ya jinsia zote katika masuala ya mazingira na majukumu ya kila jinsia. Wanyonyi (2009) alihakiki riwaya ya Nyuso Za Mwanamke ya S. Ahmed Mohammed. Katika kazi yake alishughulikia matumizi ya lugha kama vile lugha ya kitamathali, tasfida, ishara za uashiriaji na maswali balagha. Hata hivyo kazi yake alielekeza katika mtazamo wa saikolojia-changanuzi, utafiti wetu uliangalia mbinu mahususi za lugha alizozitumia mwandishi kuwakilisha maudhui ya 
mazingira katika riwaya za Tikitimaji na $M \operatorname{simu} W a$ Kipepeo huku tukiegemea nadharia za uhalisia na fasihi mazingira.

Riwaya ya pili walioichambua Nixon na Ronald (2014) katika makala yao ni riwaya ya Mohamed, Babu Alipofufuka (2001). Ni riwaya inayoonyesha athari ya uharibifu wa mazingira. Mwandishi anatumia lugha rahisi na yenye mvuto kuelezea athari ya miale ya jua kwenye utosi wa binadamu. Ingawa wataalamu wa kisayansi wanaelezea kuhusu ongezeko la joto na hata kutupa idadi kamili kwenye vipimo, fasihi inatumia tu lugha ya maelezo kuwasilisha suala hili. Riwaya ya Bustani ya Edeni ni riwaya ya tatu waliyoshughulikia na pia kuangazia masuala ya mazingira. $\mathrm{Ni}$ riwaya inayoonyesha mazingira ya jana, leo na baadaye yalivyoghasiwa na tamaa ya binadamu, uchafu unajikunjua na usafi unajikunja, maji hayanyweki na hewa haivutiki; hii yote ni kutokana na pupa ya maendeleo. Utafiti wa Nixon na Ronald unatusaidia kubainisha matendo ya binadamu katika kusababisha majanga ya kimazingira. Hata hivyo utafiti huu haujaangalia riwaya ya Tikitimaji kama riwaya ya kimazingira. Basi utafiti huu unajaza pengo hilo katika kuangazia riwaya hiyo ikiwa inatosha kuwa riwaya ya kimazingira.

Sabula (2014) anaangalia namna mwanamke anahusika katika uhifadhi wa mazingira katika himaya zilizotawaliwa na ubabe-dume. Utafiti wetu unaangalia jinsia zote na matendo yao kuhusiana na ulimwengu asilia. Utafiti wetu unanuia kuangalia jinsi mwandisha amesawiri wahusika wa jinsia zote katika kuwasilisha masuala ya mazingira.

Nwagbara (2010), katika utafiti wake kuhusu uharibifu wa mazingira nchini Nigeria, anaeleza kuwa waandishi nchini Nigeria walitumia fasihi kuhamasisha jamii kuhusu uharibifu wa mazingira na athari yake kwa maisha ya binadamu. Nwagbara anaangazia mashairi ya Ojaide ya Delta Blues and Home Songs (1998) na Daydreams of Ants and Other Poems (1997) ambayo madhumuni yake ni kuelezea uharibifu uliofanyiwa mazingira katika eneo la Delta nchini Nigeria. Hata hivyo utafiti huu unaangalia riwaya ya Tikitimaji. Utafiti huu unanuia kutathimini iwapo riwaya hizi teule zinaweza kuhamasisha umma kuhusu majanga ya kimazingira. Kwa kawaida riwaya huwa utungo mrefu ambao husimulia matukio kwa kina. Iwapo tungo za shairi ziliweza kutumiwa kuwasilisha masuala haya ya mazingira basi riwaya huwa na nafasi ipi?

Kavila (2013) katika mada yake alibainisha uhusiano wa uchafuzi wa mazingira na uhifadhi katika mazingira ya kiuchumi na kisiasa nchini Kenya. Utafiti huu haukushughulikia mifumo ya kijamii na michango yao katika uchafuzi wa mazingira mwanya ambao utafiti wetu unaziba. Utafiti wetu uliangalia masuala ya mazingira, kuanzia kwa uasilia wa ulimwengu asilia na maliasili hadi kwa uchafuzi na juhudi za kuhifadhi.

$\mathrm{Wu}$ (2016) alichambua riwaya za Kinigeria zifuatazo: Things Fall Apart (1958) ya Achebe, The Famised Road (1991) ya Okri, Burning Grass (1962) ya Kwensi, The Voice (1964) ya Okara, Sozaboy (1985) ya Saro-Wiwa na Oil On Water (2010) ya Habila. Katika makala haya, Wu (2016) anabainisha majanga ya kimazingira yanayosababishwa na ufukuzi wa maliasili na Wachina kwa hadaa za maendeleo na utandawazi. Utafiti wetu unaangalia riwaya za Wamitila na jinsi alivyowasilisha masuala ya mazingira kwa jumla, iwapo alifaulu kuwasilisha majanga ya kimazingira na njia mwafaka za kukabiliana na majanga hayo bila kuhusisha utafiti wa kisayansi bali kupitia usanii wa lugha.

Baek, Cho na Koo (2009) katika makala yao wanamnukuu Dean (2002) aliyesema kuwa uwazi wa kibiashara wa kimataifa umechangia pakubwa katika uharibifu wa mazingira. Sheria zilizowekwa ni legevu na haziwezi kudhibiti wafanyibiashara ambao azma yao ni kupata faida tu. Makala haya yanazungumzia nafasi ya utandawazi katika uharibifu wa mazingira. Utafiti wetu uliangalia jinsi utandawazi huchangia uharibifu wa mazingira, pia ulinuia kuonyesha njia mwafaka za kukabiliana na utandawazi ili kuhifadhi mazingira. Makala haya yalitufaa katika utafiti wetu katika kuangalia jinsi wahusika wanavyochangia uharibifu wa mazingira kiuchumi.

Utafiti uliofanywa na Arege katika mkusanyiko wa kazi za Adamson na Slovic (2009), unasema kuwa waandishi wa Kiswahili kama vile wa Mberia (2001) katika mkusanyiko wake wa mashairi ya Bara Jingine haswa katika mashairi ya: Jinamizi, Nieleze, Ngoi na waimbaji, Ngao na Thagicu, Mimi mto Nairobi, Okidi apiga Ukelele na Bwawa la 
Ithanje, anazungumzia suala la uchafuzi wa mazingira. Pia mashairi ya Kezilahabi (1974) katika diwani ya Kichomi kwenye shairi mahususi la Uvuaji samaki Victoria. Shairi hili linaelezea athari ya kuvua samaki kupita kiwango kinachostahili. Said Ahmed pia amejifunga kibwebwe katika uandishi huu wa kiekolojia katika mashairi yake ya Wawapi, shairi ambalo linazungumzia kupotea kwa ndege asilia. Utafiti wetu unanuia kubainisha iwapo mwandishi Wamitila katika riwaya zake hizi mbili; Tikitimaji na Msimu Wa Vipepeo ameshughulikia ipasavyo masuala ya mazingira. Je riwaya zake zitaweza kuitwa za kimazingira? Je ni mwandishi wa fasihi ambaye anaoanisha fasihi na mazingira? Hizi ndizo mianya ambazo utafiti huu unanuia kuziba. Pia tulinuia kuonyesha jinsi mwandishi huyu ameonyesha masuala mbalimbali ya mazingira kama vile uhusiano wa binadamu na mazingira athari ya matendo ya binadamu kwa ekolojia na uwezo wa ekolojia kustahimili matendo haya kupitia kwa riwaya hizi mbili teule za Wamitila.

\section{KIUNZI CHA NADHARIA}

Utafiti ulitumia nadharia ya uhalisia na nadharia ya fasihi mazingira. Vipengele katika nadharia hizi mbili ndizo zilioongoza utafiti kuthibitisha nadharia tete. Vipengele nne katika nadharia ya uhalisia vilituafiki ilihali katika nadharia ya fasihi mazingira vipengele vyote vilijumuishwa.

\section{Nadharia ya Uhalisia}

Nadharia ya uhalisia iliwekewa msingi na Hegel (1979), katika kitabu chake kilichojulikana kama Introduction to Aesthetics. Alipendekeza matumizi ya neno "uhalisia" kuelezea kazi za kifasihi ambazo huwasilisha ulimwengu na maisha ya kijamii ambako kuna wahusika au mhusika anayetenda mambo kwa jinsi inayochochewa waziwazi na hali aliyomo. Nadharia hii iliasisiwa karne ya kumi na tisa na kufafanuliwa zaidi na Groffman (1952).

Kwa mujibu wa Ntarangwi (2004), nadharia hii upana wake unamaanisha uwakilishi wa uhalisi wa mambo katika fasihi. Nadharia hii ilizuka katika karne ya kumi na tisa ili kupinga mkondo wa kiulimbwende. Mhakiki wa kihalisia anaamini katika matokeo ya mambo na ukweli na ukweli anaouzingatia ni ule ambao unaweza kuonekana na kuthibitiwa na tajriba. Nadharia hii hupembenua namna mwandishi alivyodhihirisha uhalisia wa mambo kupitia maudhui aliyoyazingatia. Msanii anapaswa kusawiri wahusika, matukio na mandhari yanayokubalika na kuaminika katika jamii. Ufahamu wa mazingira na maisha anayoyalenga mtunzi ni nguzo muhimu kwa mhakiki wa kihaliseme ukweli, iwe aminifu na ionyeshe hali halisi ya mazingira. Nadharia hii inapendekeza ujenzi wa wahusika wanaolingana na hali halisi na maisha yaani awe mhusika wa sura ileile sawa ya maisha ya kawaida kwani sanaa inafaa kueleza ukweli na inafaa kueleweka.

Dhana ya uhalisia hutumika kuakisi sifa za kimsingi zinazohusishwa na mazingira. Kama asemavyo Wamitila (2007), uhalisia unahusishwa na kipindi maalum kihistoria katika jamii za Ulaya. Kipindi hiki kinahusishwa na waandishi kama vile Balzac, Stendhal na Flaubert. Waandishi hawa wanasisitiza maelezo ya kikweli katika kazi zao na waliamini kuwa dhima ya mwandishi ni kuonyesha ukweli jinsi ulivyo. Fasihi yoyote lazima ionekane kama kioo cha jamii. Hivyo basi ni lazima jamii iweze kujitazama na kujiona kupitia fasihi.

Utafiti huu uliongozwa na mihimili ifuatayo ya nadharia hii:

a) Kazi ya msanii inapasa kuwasilisha ukweli kama ulivyo katika mazingira yake. Mhimili huu unatuonyesha jinsi maudhui ya mazingira yanavyojibainisha kihalisia kupitia mitagusano ya wahusika..

b) Msanii anaangalia matatizo na kuchunguza chanzo chake. Anachunguza mabadiliko ambayo hutokea kwa kipindi cha kihistoria na kuyatumia kuyasawiri wahusika wake. Mhimili huu unatusaidia kubainisha vyanzo vya mabadiliko ya mazingira kihistoria pamoja na mabadiliko ya wahusika katika vipindi mbalimbali vya kihistoria kwa mujibu wa ploti ya riwaya.

c) Wahusika ni vielelezo yakini vya binadamu wa kawaida kwa vile wao hutumia lugha ya kawaida kumwezesha mwanadamu kujifafanulia uwezo wake wa kutenda mambo na mtazamo wake kuhusu maisha. Mhimili huu unatusaidia kuonyesha kuwa wahusika kwenye riwaya hizi wanaathiriwa na yale wanayoyapitia. Nadharia ya Fasihi Mazingira 
Fasihi mazingira ni nadharia ambayo ina matapo katika fasihi ekolojia Kiafrika, Kimarekani na kifeministi huku ikihusisha mikabala mingine ya uhakiki katika fasihi ya Kiswahili kama vile ufeministi, umaksi na mkabala wa kibaada ukoloni. Nadharia hii inaangalia uhusiano wa fasihi na mazingira asilia. Barry (1995) anasema kuwa neno fasihi mazingira uasilia wake umehusishwa na Ruckert (1978) katika makala yake ambayo iliendelea kukua hadi miaka ya 1989 katika mkutano wake wa fasihi mazingira (World Literature Association) huko Cour d'Alene. Glotfelty na Harold(1996) walipochanganua upya neno hili na kueleza kuwa ni usomaji wa waandishi wa mazingira, dhana ambayo iliungwa mkono katika mkutano wa "Western Literature Association" na Glen Love, na kufafanua kuwa fasihi mazingira ni uhusiano wa fasihi na mazingira. Usomaji huu huchukua mtazamo wa kiardhi kama ilivyo tu ufeministi ambao huchukuwa mtazamo wa kijinsia na umaksi ambao huchukuwa mtazamo wa kimatabaka na uchumi.

Fasihi mazingira hujihusisha na matendo ya binadamu, uhusiano wa binadamu na vitu vilivyo na uhai na visivyo na uhai. Pia nadharia ii hii huangalia uchangamano wa uhusiano kati ya maliasili na utamaduni. Rigby (2007) anaeleza kuwa nadharia hii ya fasihi ya ekolojia imejitokeza kubadili hali halisi ya uharibifu wa ekolojia kwa kuchanganua uamilifu wa kimaudhui, kisanii, kijamii, kihistoria, kiitikadi na kimbinu katika ekolojia asilia au vipengele vyake kama vinavyohusishwa katika fasihi. Nadharia hii ilitokea katika mikondo miwili, wa kwanza na wa pili. Mkondo wa kwanza ulitokea miaka ya themanini kuelekea miaka ya tisini. Vigogo wa mtazamo huu wanadai kwamba uandishi wa fasihi ulenge ekolojia na dhana za ekolojia. Unapoangalia majanga za ekolojia katika karne hii huku ikibaini kuwa sayansi imejihusisha sana na masuala ya mazingira kwa hivyo wakati umewadia wa fasihi kujihusisha kikamilifu na masuala haya kwani fasihi huingiliana sana na utamaduni wa maisha ya binadamu.

Katika mkondo wa pili, Greg(2004) anaangalia mipaka iliyoko ya ubinadamu na uso ubinadamu. Pia alifasiri upya neno mazingira kumaanisha maliasili. Kama asemavyo Beull (1995), ni kutokana na ufasiri ndipo palipoibuka makongamano kuhusu haki ya mazingira asilia. Kwa mujibu wa Glotfelty na Harold (1996), Greenblatt (1992), Beull (1995) nadharia hii huangalia mielekeo ya binadamu kuhusu mazingira, huangalia matumizi ya dhana za ekolojia katika kazi za kifasihi, hujihusisha na nguvu za kibinadamu na lugha ya binadamu kuhusiana na mazingira, huangalia mitindo ya lugha na jinsi mitindo hiyo huathiri namna binadamu anavyotagusana na mazingira, nadharia hii huchukulia kwamba mazingira na utamaduni hushirikiana katika ujenzi wa lugha na fasihi, binadamu hawezi kuishi bila ekolojia lakini ekolojia huweza kuishi bila binadamu, huangalia jinsi waandishi huwakilisha masuala ya mazingira na jinsi kazi hiyo huathiri mielekeo ya ulimwengu katika hali halisi na mwisho huangalia uwezo wa lugha katika kuwasilisha masuala ya mazingira.

Nadharia hizi mbili zinachangia katika kuthibitisha nadharia tete za utafiti huu. Nadharia ya fasihi mazingira imetumiwa kuchanganua na kubainisha dhana za ekolojia kama vile viwango vya mpangilio wa kibiolojia, viumbe asilia, uwezekano wa ekolojia kustahamili matendo ya uchafuzi na kurejelea hali asilia pamoja na mitindo ya lugha iiliyotumika kuwasilishia dhana hizi za ekolojia. Huku nadharia ya uhalisia ikinisaidia ikitumiwa kubainisha ukweli wa masuala ya mazingira katika kazi za fasihi.

\section{MBINU ZA UTAFITI}

Muundo wa utafiti ulikuwa wa kiuchanganuzi. Huu ni muundo ambao mtafiti hutumia data ambayo iko tayari kisha kuichanganua kulingana na mada husika. Riwaya teule za Wamitila, Tikitimaji na Msimu Wa Vipepeo tayari zimekwisha andikwa mbinu ambayo ilituafiki katika utafiti wetu. Eneo kithematiki ni vitabu vya Wamitila. Kundi lengwa ni riwaya za Wamitila. Vitabu vyake viwili viliteuliwa kwa sababu mwandishi huyu anaonyesha ari katika uwanja wa fasihi mazingira kupitia makala yake ya "Place and placelessness in Kiswahili setting. The role of setting in Kiswahili creative writing." Riwaya za Tikitimaji (2013) na Msimu Wa Vipepeo (2006) ndizo matini zilizochaguliwa kimakusudi na mtafiti. Mtafiti alifikia sampuli hii baada ya kusoma riwaya za Wamitila na kuteua kimakusudi riwaya hizi kwa sababu riwaya hizi zinazungumzia masuala ya 
mazingira. Data ilipatikana kutoka kwa matini hizi kimakusudi yaani kubainisha maudhui hayo kutokana na wahusika na pia mitindo na kuyahusisha na uhalisia ikitathimini kufaulu kwa mwandishi. Njia kuu ya kukusanya data ilikuwa ya uchunzaji ambapo kifaa cha kukusanya data kilikuwa ni orodha ya uchunzaji. Sehemu zenye kuashiria maudhui ya mazingira ikijumuisha mtindo na wahusika. Pia mbinu ya usomaji makini ilihusishwa. Mbinu ya uchanganuzi wa yaliyomo ilitumiwa kutathmini na kueleza kimfumo yaliyomo katika data ya mwandishi au maongezi. Hali ambayo ilituwezesha kuchunguza nafasi iliyopewa masuala ya mazingira katika riwaya hizi mbili teule.

\section{UWASILISHAJI WA MATOKEO}

Matokeo ya utafiti yaliwasilishwa kwa njia ya maelezo. Maelezo hayo yanahusu jinsi maudhui ya mazingira yalivyosawiriwa katika riwaya ya Tikitimaji na Msimu Wa Vipepeo. Kwa njia ya maelezo, mtafiti aliorodhesha mbinu za lugha zilizotumiwa na mwandishi kudokeza maudhui ya mazingira. Pia mtafiti alishughulikia wahusika jinsi walivyosawiriwa na mwandishi ili kukuza maudhui haya ya mazingira. Mifano ilinukuliwa ili kuelezea mbinu alizozitumia mwandishi kubainisha maudhui ya mazingira kwa mujibu wa malengo ya utafiti na mihimili ya nadharia mbili zilizoteuliwa.

\section{Madhumuni ya kwanza Kuhusu Wahusika}

Katika utafiti huu imebainika kuwa fasihi ina nafasi kubwa katika kuwasilisha masuala ya mazingira. Mwandishi Wamitila amewasilisha maudhui ya mazingira ipasavyo kupitia vipengele vya fasihi. Vipengele hivi vya fasihi ni pamoja na wahusika, mandhari, ploti na mtindo. Katika kipengele cha wahusika, mwandishi amewapanga wahusika kimakusudi kulingana na majukumu yao. Kunao wahusika watetezi na wakombozi wa mazingira. Wahusika hawa wamesawiriwa katika miktadha ya kijamii, kisiasa na kiuchumi. Katika riwaya ya Tikitimaji,wahusika Zinji, Sapna, Maya na Kombo wamesawiriwa kijamii na kiuchumi katika kuelezea uhalisia wa masuala ya mazingira. Katika safari yao kuelekea Tikitimaji, mwandishi anatupa picha halisi ya maeneo wanamopitia kijiogirafia na kutuonyesha mabadiliko ya anga pamoja na bioanuai. Mwandishi analinganisha na kulinganua mazingira walimotoka na yale walimoelekea. Kupitia mitagusano yao na wahusika wengine, mwandishi anadhihirisha mihemko na mielekeo ya binadamu kuhusu mazingira. Wahusika wamepangwa kulingana na majukumu yao. Kuna wale watetezi na wakombozi wa mazingira kama kina Kanda, Mzee Kitali katika riwaya ya Msimu Wa Vipepeo na Zinji, Sapna, Maya na Kombo katika riwaya ya Tikitimaji. Wahusika hawa wamesawiriwa kijamii, kisiasa na kiuchumi.

Katika mfumo wa kijamii wahusika wanapaswa kushirikiana ili kuhifadhi na kukemea wachafuzi wa mazingira. Nadharia hii imethibitika kupitia wahusika Zinji, Sapna, Maya, Kombo na Mzee Jududi katika riwaya ya Tikitimaji na Kanda, Mkurutu na Fabio Mkalla katika riwaya ya Msimu Wa Vipepeo. Wahusika katika riwaya hizi mbili wamesawiriwa wakitagusana na mazingira katika hali za kijamii, kisiasa na kiuchumi. Katika riwaya ya Msimu Wa Vipepeo, Mkurutu anatagusana na mazingira kupitia kilimo lakini ananuia kufaidika kisiasa, ambapo anatumaia cheo alichopewa na wananchi kuhujumu mazingira.

Ingawa yeye ni Waziri wa Mazingira na Utalii hajishughulishi hata kidogo na mijadala ibuka kuhusiana na visababishi vya uchafuzi wa mazingira. Tetesi zinapoibuka kwamba ipo meli iliyokuwa inamwaga mafuta kwenye mto, hali iliyotishia viumbe wa baharini, yeye anafutilia mbali zile tetesi. Kwa upande mwingine, Kanda anaendeleza shughuli za uhifadhi wa mazingira katika riwaya hii ya Msimu Wa Vipepeo. Ingawa hana kipato kikubwa anajitolea kuhifadhi mazingira na kuwapa nasaha waliochafua mazingira. Anawahimiza watu hao wafuate ushauri unaotolewa na vyama vya mazingira.

Katika riwaya ya Tikitimaji wahusika Zinji, Sapna, Maya na Kombo ni vielelezo vya wahusika ambao wanaendeleza shughuli za uhifadhi wa mazingira. Wanaungana kwa pamoja na kuunda kundi la vuguvugu la kulinda mazingira. Hawa ni wahusika vielelezo ambao wanathibitisha kuwa jinsi wanasiasa wanavyotengeneza vuguvugu la kisiasa, basi hata mazingira yastahili kupiganiwa. Ni wahusika waliochanganywa jinsia na hakuna jinsia iliyopewa jukumu la kuhifadhi mazingira pekee yake bali kila mwanajamii amepewa jukumu hili bila kubagua jinsia. 
Mhusika Ndugu Kiwiro amesimamia masuala ya usafi ila afanyacho ni kinyume kabisa na cheo alichopewa. Yeye ni mhusika ambaye kabisa anadidimiza shughuli za uhifadhi wa mazingira. Kwake mazingira si muhimu; cha muhimu ni kile kinachotokana na mazingira. Anasema kuwa haijalishi kama binadamu atatia kitanzi mazingira au hata kutoboa utumbo wake; kwake kilicho muhimu ni faida itokanayo na mazingira. Ni mhusika anayeamini kuwa mazingira hayapaswi kuhifadhiwa kwa ajili ya urembo. Ingawa yeye ni msomi, hana maarifa asilia kutokana na umuhimu wa uhifadhi wa mazingira kama mzee Jududi ambaye hana kisomo lakini anayo maarifa asilia kuhusu umuhimu wa kuhifadhi mazingira.

\section{Madhumuni ya pili kuhusu mtindo}

Madhumuni yetu ya pili yalihusu maelezo kuhusu lugha na mitindo iliyotumika kuwasilisha maudhui ya mazingira. Mbinu ya takriri imetumika kwa wingi kwa mfano katika riwaya ya Tikitimaji mwandishi amerudia maneno haya hapa na pale kutoa picha halisi ya mimea asilia katika mandhari aliyokuwa akisimulia. Maneno yaya haya pia yamerudiwa katika kurasa zinazofutia kuelezea uchache wa viumbe asili. Aliteua mbinu hii ya takriri kimakusudi kusisitiza na kuonya watu kuhusu uchafuzi wa mazingira pamoja na athari za kutohifadhi mazingira.

Katika riwaya ya Msimu Wa Vipepeo, mwandishi ametumia mbinu rejeshi kimakusudi kuelezea mabadiliko ya mazingira kihistoria. Pia mbinu hii anaitumia kuelezea chanzo cha uchafuzi wa mazingira. Kupitia mbinu hii mhusika Kanda anatueleza chanzo cha kuwepo kwa moshi uliotanda kote jijini. Anaeleza kuwa moshi huo ulitokana na kupuuzwa kwa ushauri uliopewa na wanachama wa walinda mazingira wa kutumia aina ya mafuta ya gari ambayo haina salfa. Ubinafsi wa wenye viwanda nao uliwafanya kupuuza ushauri huo.

\section{HITIMISHO}

Mwandishi amewasilisha masuala ya uchafuzi wa mazingira kama vile: umwagaji wa uchafu kutoka viwandani kwenye mito, ukataji wa miti, ujenzi wa majumba karibu na fuo za bahari, uhasama kati ya wanyama na binadamu, faida za wanyama, ndege, mlima na misitu kwa wanadamu, changamoto zinazohusiana na ukombozi wa mazingira na nafasi ya wajamii wote kuhusiana na suala la kuajibikia mazingira. Pia mwandishi ametoa suluhisho kuhusiana na maeneo ambayo tayari yamechafuliwa. Anashinikiza ushirikiano wa kila mmoja katika kuhifadhi eneo anamosakini. Mwandishi anafutilia mbali dhana potovu kuwa uchafuzi wa mazingira unafanyika tu mijini. Anasema kuwa uchafuzi wa mazingira ni jambo lililoenea kila pahali hata mashambani. Mara nyingi watu wengi hukimbilia vijijini ili kupata hewa safi. Mwandishi amependekeza njia mwafaka za kukabiliana na hewa chafu kwenye miji yetu ambayo huwa na msongamano wa magari.

Anaonya kuwa suala la uhifadhi wa mazingira huenda likawa suala ngumu iwapo wanadamu hawatakuwa na moyo wa kujitolea na kuwaza kama mlima. Kupitia matini hii, mwandishi ana lengo moja ambalo ni kusitisha uharibifu wa mazingira. Hii ndiyo maana inayojitokeza katika riwaya hizi mbili; amehusisha vyama kama vile cha Enviromental Climate Watch katika riwaya ya Msimu Wa Vipepeo na chama cha WAZALIMA katika riwaya ya Tikitimaji.

Ingawa kwenye riwaya ya Msimu Wa Vipepeo sheria zile hazikutumika kama walivyopendekeza hawa walinda mazingira, wahusika wanajutia kwa kutozitumia na wakabaki wakijutia. Hivyo msomaji ameachiwa uamuzi wa kuzifuata ili kuepuka majanga ya kimazingira au kujipata kwenye simanzi ileile. Pia anawahamasisha wanajamii kuhusu uhifadhi wa mazingira kuwa ni wajibu wa kila mwanajamii kupitia vipengele vya fasihi ambayo ni wahusika, mtindo na mandhari.

Masuala ya mazingira yanaendelea kuzungumziwa katika nyanja mbalimbali. Fasihi nayo haijaachwa nyuma katika mjadala huu. Fasihi ya Kiswahili inayo uwezo wa kuyamulika masuala ya kiekolojia kama yanavyoonekana katika jamii. Ni muhimu isemwe bayana kuwa vipengele vyote vya fasihi havikushughulikiwa katika utafiti huu. Kwa ajili hii mtafiti anapendekeza utafiti wa baadaye utumie nadharia nyingine kama vile nadharia ya kibaada ukoloni katika kuhakiki masuala ya kimazingira kwani ukoloni umechangia pakubwa katika uchafuzi wa mazingira. 


\section{MAREJELEO}

Adamson, J., \& Slovic, S. (2009). Guest editors' introduction the shoulders we stand on: an introduction to ethnicity and ecocriticism. Melus, 34(2), 5-24.

Baek, J., Cho, Y., \& Koo, W. W. (2009). The environmental consequences of globalization: A country-specific time-series analysis. Ecological Economics, 68(8-9), 2255-2264.

Barry, P. (1995). Beginning Theory: An introduction to literary and cultural theory. Manchester: Manchester University Press.

Beull, L. (1995). The Environmental Imagination: Thoreau, nature writing, and the formation of American culture. Cambridge University Press.

Cresswell, J. (2009). Research design: Quantitative, qualitative mixed-method approaches. Los Angels: SAGE publication.

Glotfelty, C. \& Harold, F. (1996). The ecocriticism reader: Landmarks in Literary Ecology. Athens: University of Georgia Press.

Greg, G. (2004). Ecocriticism. London and New York: Routledge.

Greenblatt, S. (1992). Marvellous Possessions: The wonder of the New World: Chicago University Press.

IPCC. (2013). Climate change 2013:The Physical Science Basis. Contribution of working group 1 to the Fifth Assessment Report of the New York, NY, USA: Cambridge University Press.Available:

http://www.climatechange2013.org/images/up loads/WGS_AR5_SPM_Bronchure.pdf.

IPCC (2014).Climate Change 2014: Mitigation of climate of Climate Change Working Group III Contribution to the Fifth Assessment Report of the Intergovernmental Panel on Climate change. Cambridge University Press; New York: 2014 chap AnnexII.2 [Google Scholar]

Kavila, F. S. (2013). The Theme of Environmental Conservation in Different Colours and
Terrorists of the Aberdare by Ng'ang'a Mbugua. Doctoral dissertation. University of Nairobi.

Mohamed, S. A. (2001). Babu Alipofufuka. Nairobi: Jomo Kenyatta Foundation.

Moore, N. (2008). Eco/feminism, non-violence and the Future of Feminism. International Journal of Politics, 10(3), 282-298.

Nixon, N. S., \& Ronald, O. N. (2014). Literary environmentalism: An ecocritique of Kiswahili literature. International Journal of Ecology and Ecosolution, 1(2), 29-41.

Njogu, K \& Chimera, R. (1999). Ufundishaji wa fasihi: Nadharia na mbinu. Nairobi: Jomo Kenyatta Foundation.

Ntarangwi, M. (2004). Uhakiki wa kazi za fasihi. Rock Island: Augustana college:.

Nwagbara, U. (2010). Poetics of Resistance: Ecocritical reading of Ojaide's poems "Delta Blues and Home Songs and Daydream of Ants and other poems." African study monographs, 31 (1), 17-30.

Reuveny, R. (2007). Climate Change-induced migration and violent conflict. Political geography 26, 656-67.

Rigby, K. (2007). Ecocriticism: introducing ecocriticism in the twenty-first century. Edinburgh: UP.

Rueckert, W. (1978). Literature and Ecology: An Experiment in Ecocriticism. Athens: University of Georgia Press

Sabula, M. A. (2014). Nafasi ya wanawake katika uhifadhi wa mazingira katika riwaya ya Nakuru to: mtazamo wa fasihi ya ufeministi wa kiekolojia. Chuo Kikuu cha Nairobi

Vasquez, A. (1983). Art and Society in Essays in Marxist Aesthetics. New York: Monthly Review Press.

UNFCC. 2009. Decisionx.CP.15Methodological guidance for activities relating to reducing emissions from deforestation and forest 
degradation and the role of conservation, sustainable management forests and enhancements of forests carbon stocks in developing countries.

FCC/CP/2009/11Add.1,decision4/CP.15.

Retrieved from http://unfccc.int/files/na/application/pdf/cop15 ddc auv.pdf. Accessed 07/08/2017.

Wa Mberia, K. (2001). Bara Jingine. Nairobi: Mariba publications.

Wafula, R. M., \& Njogu, K. (2007). Nadharia za uhakiki wa fasihi. Nairobi, KE: Jomo Kenyatta Foundation.

Wamitila, K. W. (2004). Kichocheo cha fasihi simulizi na Andishi. Focus publishers.

Wamitila, K. W. (2006). Msimu wa vipepeo. Nairobi: Vide-Muwa Publishers

Wamitila, K. W. (2013). Tikitimaji. Nairobi: Vide Muwa Publishers.

Wanyonyi, A. (2009). Sintaksia, italiki, lugha ya kitamathali, Tasfida, Ishara za uwashiriaji na masuala balagha katika riwaya ya Nyuso Za Mwanamke. Tasnifu ya Uzamili (haijachapishwa). Chuo kikuu cha Nairobi.

Wu, C. C. (2016). Towards an Africa-Focused Ecocriticism: The Case of Nigeria. Doctoral dissertation. Chuo Kikuu cha Nevada, Reno. 